\title{
Sepsis y choque séptico en los servicios de urgencias de México: estudio multicéntrico de prevalencia puntual
}

\author{
Luis A. Gorordo-Delsol,,2* Graciela Merinos-Sánchez,, ${ }^{2,3}$ Ricardo A. Estrada-Escobar, ${ }^{3}$ \\ Nikolett I. Medveczky-Ordoñez, ${ }^{1}$ Marcos A. Amezcua-Gutiérrez, ${ }^{1}$ Ma. Angélica Morales-Segura ${ }^{4}$ y \\ Silvia E. Uribe-Moya

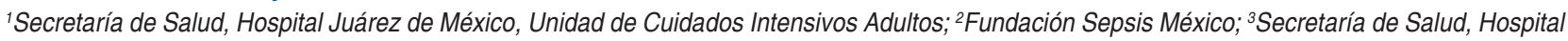 \\ General de México "Dr. Eduardo Liceaga", Servicio de Urgencias en Adultos; ${ }^{4}$ Instituto Politécnico Nacional, Escuela Superior de Medicina, Sección \\ de Estudios de Posgrado e Investigación; ${ }^{5}$ Secretaría de Salud, Hospital Juárez de México, Servicio de Urgencias. Ciudad de México, México
}

\section{Resumen}

Introducción: La sepsis es una de las principales causas de consulta en los servicios de urgencias médicas. Objetivo: Identificar la prevalencia de la sepsis en servicios de urgencias médicas de México. Método: Estudio transversal con seguimiento a 30 días de los pacientes con diagnóstico de sepsis o choque séptico; se analizaron las variables demográficas, el manejo y los desenlaces. Resultados: En 68 servicios de urgencias médicas analizados se atendió a 2379 pacientes, de los cuales 307 presentaron sepsis. La prevalencia de la sepsis fue de $12.9 \%$, con mortalidad global de $16.93 \%$, que en los casos de sepsis fue de $9.39 \%$ y en los de choque séptico, de $65.85 \%$; no se identificaron diferencias significativas en las variables demográficas o tipo de hospital. Se observó balance hídrico alto en las primeras tres horas y falta de apego a las recomendaciones internacionales de reanimación superior en los pacientes que fallecieron. Conclusiones: Se encontró alta prevalencia de la sepsis en los servicios de urgencias médicas mexicanos. La mortalidad de los pacientes con choque séptico fue similar e, incluso, mayor a la reportada internacionalmente.

PALABRAS CLAVE: Sepsis. Servicio de emergencias hospitalario. Epidemiología.

\section{Sepsis and septic shock in emergency departments of Mexico: a multicenter point- prevalence study}

\begin{abstract}
Introduction: Sepsis is one of the main reasons for consultation at emergency departments. Objective: To identify the prevalence of sepsis in emergency departments of Mexico. Method: Cross-sectional study with a 30-day follow-up of patients diagnosed with sepsis or septic shock; demographic variables, management and outcomes were analyzed. Results: In 68 emergency departments analyzed, 2379 patients were attended to, out of whom 307 had sepsis. The prevalence of sepsis was $12.9 \%$, and overall mortality was $16.93 \%$, which in the cases of sepsis was $9.39 \%$, and in those of septic shock, 65.85 $\%$; no significant differences were identified in demographic variables or type of hospital. A significantly higher fluid balance was observed within the first three hours in those patients who died, as well as a lack of adherence to international resuscitation recommendations. Conclusions: $A$ high prevalence of sepsis was found in Mexican emergency departments. Mortality of patients with septic shock was similar and even higher than that internationally reported.
\end{abstract}

KEY WORDS: Sepsis. Hospital emergency department. Epidemiology.

Correspondencia:

*Luis A. Gorordo-Delsol

E-mail: luis.gorordodelsol@icloud.com

0016-3813/๑ 2020 Academia Nacional de Medicina de México, A.C. Publicado por Permanyer. Este es un artículo open access bajo la licencia CC BY-NC-ND (http://creativecommons.org/licenses/by-nc-nd/4.0/).
Fecha de recepción: 16-08-2019

Fecha de aceptación: 06-07-2020

Gac Med Mex. 2020;156:495-501

Disponible en PubMed

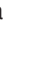




\section{Introducción}

La sepsis se define como la falla orgánica potencialmente mortal causada por la respuesta desproporcionada del organismo ante una infección. ${ }^{1}$ Los criterios clínicos para el diagnóstico de sepsis son presencia de una infección y disfunción orgánica o exacerbación de una falla orgánica crónica. ${ }^{1-3}$ Se recomiendan establecer el diagnóstico de sepsis en pacientes sin falla orgánica preexistente con una puntuación $\geq 2$ del Sequential Organ Failure Assessment (SOFA) o con incremento $\geq 2$ puntos en pacientes con fallas orgánicas crónicas. ${ }^{2-4} \mathrm{El}$ choque séptico es un subtipo de sepsis que incrementa $40 \%$ la mortalidad e incluye alteraciones circulatorias con anormalidades del metabolismo celular. ${ }^{5}$

Las infecciones constituyen una de las principales causas de atención en los servicios de urgencias médicas (SUM) y en numerosas ocasiones desencadenan sepsis y choque séptico. Paradójicamente, la mayoría de los estudios se aboca al manejo de los pacientes con sepsis en las unidades de cuidados intensivos (UCl), ${ }^{6,7}$ aun cuando el manejo inicial en los SUM es clave ${ }^{6-8}$ y numerosos pacientes con choque séptico no ingresarán a las $\mathrm{UCl} .^{1}$ Comprender la relevancia de los SUM es vital para dirigir los recursos e implementar medidas de mejora de la calidad.., 10

Numerosos estudios, la mayoría en Estados Unidos, Europa y Oceanía, ${ }^{11-16}$ se enfocan en la atención en las $\mathrm{UCl}$ : un reporte reunió $735 \mathrm{UCl}$, en las cuales se registraron 88371 pacientes, $15.08 \%$ con sepsis o choque séptico; la mortalidad fue de 20 a $59 \% .^{15}$ El estudio EPIC-II indicó que $51 \%$ de los pacientes en UCl cursaba con infecciones. ${ }^{16}$ Un estudio de $18 \mathrm{UCI}$ mexicanas reportó que $85 \%$ de los ingresos se relacionó con sepsis. ${ }^{17}$ Otro estudio mexicano de $135 \mathrm{UCl}$ indicó que $27.3 \%$ de los ingresos se debió a choque séptico y que $30.4 \%$ falleció. ${ }^{18}$ Una investigación en SUM registró que aproximadamente $60 \%$ de los pacientes presentó sepsis y $10 \%$ ingresó con choque séptico. ${ }^{19}$

Los objetivos de la investigación que se describe fueron identificar la prevalencia de sepsis en SUM mexicanos y describir el apego a las recomendaciones de manejo.

\section{Método}

Estudio multicéntrico, observacional y transversal, con seguimiento a 30 días para identificar la prevalencia de sepsis y la mortalidad por esta causa en
SUM mexicanos. El estudio fue aprobado por el Comité de Investigación con registro HJM-0515/18-I; no se requirió consentimiento informado. Se siguieron las recomendaciones de la Iniciativa STROBE (Strengthening the Reporting of Observational Studies in Epidemiology). ${ }^{20}$

Los centros participantes se reclutaron por invitación de la Fundación Sepsis México, la Sociedad Mexicana de Medicina de Emergencias A. C. y la Asociación de Medicina de Emergencias y Desastres de México A. C. La promoción se llevó a cabo con anuncios en reuniones nacionales y redes sociales. La participación fue voluntaria. Cada centro colaborador recibió una copia del protocolo y los permisos para ingresar a la plataforma en la cual se trabajo; además, respondió una encuesta sobre sus características.

Se incluyeron los pacientes que ingresaron a los SUM participantes durante un periodo de 24 horas; la plataforma filtró a los pacientes conforme los criterios de inclusión: edad $\geq 18$ años, diagnóstico confirmado o presuntivo de sepsis o choque séptico según las definiciones establecidas en el The Third International Consensus Definitions for Sepsis and Septic Shock (Sepsis-3). ${ }^{2-6,10}$ Se excluyeron los pacientes referidos de otra institución y se eliminaron los pacientes con indicaciones de limitación de esfuerzo terapéutico, cuidados paliativos y egresados por alta voluntaria o trasladados a otras institución. Para evitar errores en la captura de información, la plataforma se codificó con bloqueos automáticos. El análisis estadístico fue realizado por los investigadores y las discrepancias fueron verificadas con los centros colaboradores.

El análisis descriptivo de las variables de escala se llevó a cabo con medidas de tendencia central y dispersión y el de las variables categóricas con frecuencias y porcentajes. Se usó la prueba de Kolmogorov-Smirnov para la normalidad de la curva, con la cual se obtuvieron resultados no paramétricos, y posteriormente se aplicó la prueba $U$ de Mann-Whitney. Las asociaciones entre grupos y variables se determinaron mediante $\chi^{2}$, en tanto que la comparación entre grupos se efectuó por prueba exacta de Fisher. Los factores de riesgo se midieron con razón de momios (RM) e intervalos confianza de $95 \%$ (IC $95 \%$ ); la asociación entre más de dos medias se estableció con el coeficiente de correlación de Spearman. Los resultados fueron significativos cuando el valor de $p$ fue $<0.05$. Se utilizó el programa estadístico SPSS versión 21. Los datos faltantes se abordaron mediante eliminación de casos o imputación del valor medio. 


\section{Resultados}

Se registraron 211 centros colaboradores, de los cuales 13 no eran elegibles por ser SUM especializados; de 198 SUM elegibles, 101 (47.86\%) respondieron la encuesta inicial y solo $68(32.22 \%)$ incluyeron pacientes en el estudio (Figura 1).

El día del estudio ingresaron 2379 pacientes a los SUM participantes; 307 pacientes (12.9\%) fueron diagnosticados con sepsis y 41 de ellos (13.35\%) llegaron con choque séptico. Participaron cinco hospitales privados y 63 hospitales públicos, sin diferencias en el riesgo de mortalidad por todas las causas a los 30 días (RM = 1.093, IC $95 \%=0.229-5.214$, $p=0.911)$. La edad media fue 53.6 años, sin diferencias significativas entre los supervivientes y los no supervivientes. Se registraron 172 mujeres (56.02\%); el sexo no se asoció a mayor riesgo de mortalidad a los 30 días $(\mathrm{OR}=1.314$, IC $95 \%=0.714-2.421$, $\mathrm{p}=0.380)$. El índice de masa corporal $\left(\mathrm{kg} / \mathrm{m}^{2}\right)$ promedio fue 26.7.

Se identificó una prevalencia de sepsis y choque séptico de $12.9 \%$ en la población en estudio (IC $95 \%$ = 11.62-14.11\%); de los 307 pacientes incluidos, al ingreso en 266 (86.64\%) se cumplieron los criterios de sepsis y en 41 (13.35\%) de choque séptico. La mortalidad global representó $16.93 \%, 9.39 \%$ en los pacientes con sepsis y $65.85 \%$ en los pacientes con choque séptico, lo cual se tradujo en una asociación significativa entre choque séptico y mortalidad a los 30 días, con RM = 18.5914 (IC $95 \%=8.6456$ 39.9806, $\mathrm{p}=0.0001$ ).

La infección más común fue la del tracto urinario, seguida de las neumonías adquiridas en la comunidad. La neumonía fue la única causa de sepsis que se asoció con aumento significativo de la mortalidad a los 30 días $(\mathrm{RM}=1.9429, \mathrm{IC} 95 \%=1.0085-3.7428$, $\mathrm{p}=0.0471$; (Tabla 1).

De los 307 casos, se realizó cuantificación del lactato en 64 pacientes (20.8\%). Se obtuvieron 168 cultivos (70 de sangre, 53 de orina, 19 de esputo, cinco de expectoración con trampa y 21 de otras secreciones) de 120 pacientes (39.08\%), de los cuales $19.64 \%$ fue positivo. Solo 287 pacientes recibieron antibióticos durante su estancia en los SUM; en $29.96 \%$ se iniciaron durante la primera hora (Tabla 2).

Entre los pacientes en quienes se observó un balance de líquidos $\geq 30 \mathrm{~mL} / \mathrm{kg}$ en las primeras tres horas, el total acumulado en los supervivientes y los no supervivientes fue similar (39.95 y $39.94 \mathrm{~mL} / \mathrm{kg}$,

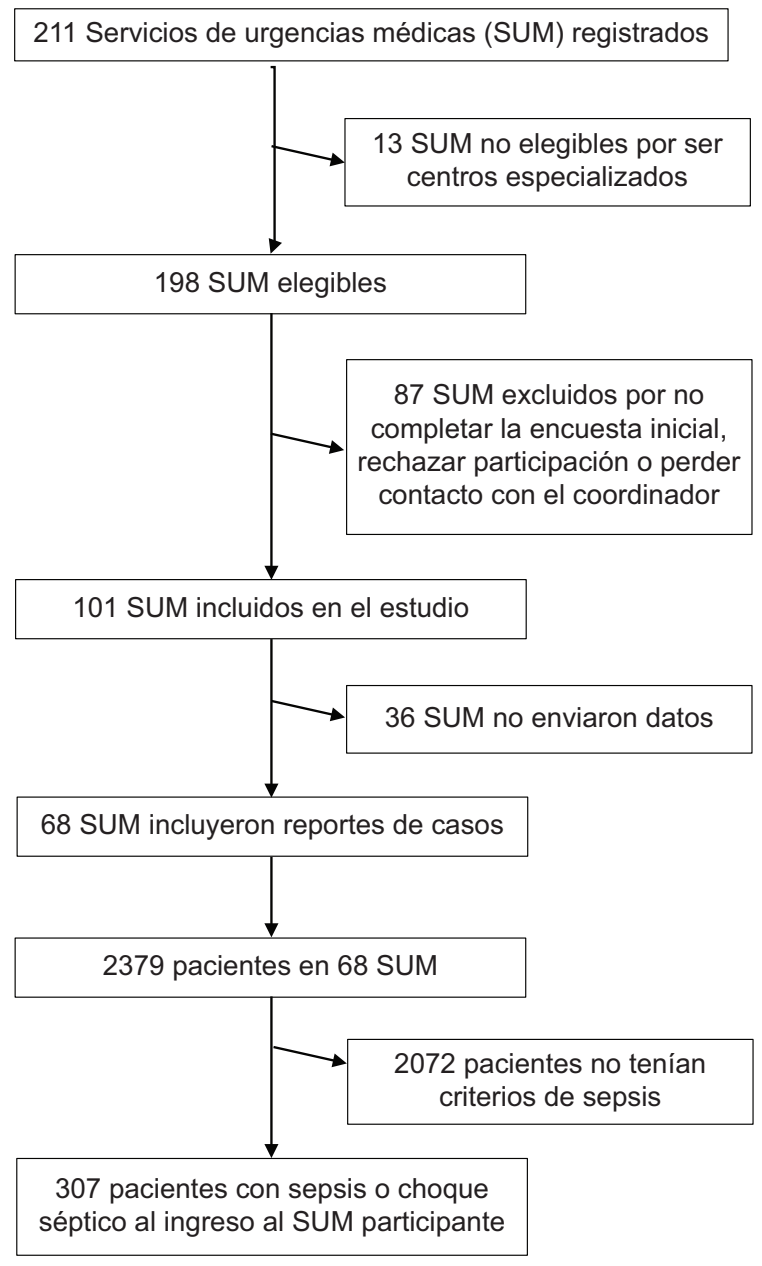

Figura 1. Flujograma de inclusión de centros participantes y pacientes.

$p=0.7263$ ); en lo pacientes en quienes el balance hídrico se mantuvo por debajo de $30 \mathrm{~mL} / \mathrm{kg}$ en las primeras tres horas, la media entre los supervivientes y no supervivientes no fue diferente $(7.74$ y $9.19 \mathrm{~mL} / \mathrm{kg}$, $p=0.7565)$. En los pacientes en quienes no se logró la meta de balance de líquidos $\geq 30 \mathrm{~mL} / \mathrm{kg}$ se observó reducción de la mortalidad a 30 días $(\mathrm{RM}=0.2820$, IC $95 \%=0.1341-0.593, p=0.001$ ), en comparación con quienes se logró; el promedio de los sobrevivientes fue significativamente menor que el de los no sobrevivientes (10.68 versus $17.4 \mathrm{~mL} / \mathrm{kg}, \mathrm{p}=0.0271$ ). El balance de líquidos acumulados en las primeras seis horas fue de 42.6 versus $62.2 \mathrm{~mL} / \mathrm{kg}(p=0.0587)$ y a las 24 horas de 35 versus $47 \mathrm{~mL} / \mathrm{kg}(p=0.0969)$.

Fallecieron cuatro de los nueve pacientes en quienes durante la primera hora se cumplieron las medidas de reanimación recomendadas ${ }^{21}$ y 48 de 298 pacientes en quienes no se cumplieron, con una asociación positiva entre no cumplir los objetivos y menor mortalidad $(\mathrm{RM}=0.240, \mathrm{IC} 95 \%=0.062-0.926$, 
Tabla 1. Datos demográficos, fuente de infección y escalas de gravedad en pacientes con sepsis atendidos en servicios de urgencias médicas

\begin{tabular}{|c|c|c|c|c|c|c|}
\hline & \multicolumn{2}{|c|}{ Todos los pacientes } & \multicolumn{2}{|c|}{ Sobrevivientes } & No sobrevivientes & $p$ \\
\hline & \multicolumn{2}{|c|}{ Media } & \multicolumn{2}{|c|}{ Media } & Media & \\
\hline Edad en años & \multicolumn{2}{|c|}{53.6} & \multicolumn{2}{|c|}{52.38} & 59.57 & 0.023 \\
\hline $\begin{array}{l}\text { Escalas de gravedad al ingreso a urgenc } \\
\text { APACHE II } \\
\text { SAPS II } \\
\text { SOFA } \\
\text { qSOFA } \\
\text { MPM-II }\end{array}$ & \multicolumn{2}{|c|}{$\begin{array}{c}10.65 \\
32.47 \\
5.22 \\
0.99 \\
15.21\end{array}$} & \multicolumn{2}{|c|}{$\begin{array}{c}9.43 \\
30.62 \\
4.82 \\
0.82 \\
12.77\end{array}$} & $\begin{array}{c}16.95 \\
42.08 \\
7.32 \\
1.78 \\
28.26\end{array}$ & $\begin{array}{l}<0.0001 \\
<0.0001 \\
<0.0001 \\
<0.0001 \\
<0.0001\end{array}$ \\
\hline & \multicolumn{2}{|c|}{$n$} & \multicolumn{2}{|c|}{$\mathrm{n}$} & $n$ & \\
\hline $\begin{array}{l}\text { Sexo } \\
\text { Femenino } \\
\text { Masculino }\end{array}$ & \multicolumn{2}{|c|}{$\begin{array}{l}172 \\
135\end{array}$} & \multicolumn{2}{|c|}{$\begin{array}{l}140 \\
115\end{array}$} & $\begin{array}{l}32 \\
20\end{array}$ & 0.380 \\
\hline IMC $\left(\mathrm{kg} / \mathrm{m}^{2}\right)$ & \multicolumn{2}{|c|}{26.7} & \multicolumn{2}{|c|}{26.8} & 26.5 & 0.6718 \\
\hline $\begin{array}{l}\text { Tipo de institución } \\
\text { Pública } \\
\text { Privada }\end{array}$ & \multicolumn{2}{|c|}{$\begin{array}{c}296 \\
11\end{array}$} & \multicolumn{2}{|c|}{$\begin{array}{c}246 \\
9\end{array}$} & $\begin{array}{c}50 \\
2\end{array}$ & 0.911 \\
\hline \multirow[t]{2}{*}{$\begin{array}{l}\text { Severidad de la sepsis } \\
\text { Sepsis } \\
\text { Choque séptico }\end{array}$} & \multicolumn{2}{|c|}{$\begin{array}{c}266 \\
41\end{array}$} & \multicolumn{2}{|c|}{$\begin{array}{c}241 \\
14\end{array}$} & $\begin{array}{l}25 \\
27\end{array}$ & $\leq 0.0001$ \\
\hline & $\mathrm{n}$ & $\%$ & $\mathrm{n}$ & $\%$ & n & $\%$ \\
\hline $\begin{array}{l}\text { Sitio de infección } \\
\text { Neumonía } \\
\text { Cirugía torácica } \\
\text { Infección intrabdominal no quirúrgica } \\
\text { Infección intrabdominal quirúrgica } \\
\text { Tracto urinario } \\
\text { Piel y tejidos blandos } \\
\text { Otras }\end{array}$ & $\begin{array}{c}68 / 307 \\
2 / 307 \\
43 / 307 \\
25 / 307 \\
97 / 307 \\
51 / 307 \\
21 / 307\end{array}$ & $\begin{array}{c}22.1 \\
0.6 \\
14 \\
8.1 \\
31.5 \\
16.6 \\
6.8\end{array}$ & $\begin{array}{c}51 / 68 \\
2 / 2 \\
37 / 43 \\
20 / 25 \\
87 / 97 \\
46 / 51 \\
12 / 21\end{array}$ & $\begin{array}{c}75 \\
100 \\
86 \\
80 \\
89.6 \\
90.1 \\
57.14\end{array}$ & $\begin{array}{c}17 / 68 \\
0 / 2 \\
6 / 43 \\
5 / 25 \\
10 / 97 \\
5 / 51 \\
9 / 21\end{array}$ & $\begin{array}{c}25 \\
0 \\
14 \\
20 \\
10.3 \\
9.8 \\
42.8\end{array}$ \\
\hline
\end{tabular}

$p=0.038)$. En cuanto a la realización de las medidas recomendadas durante las tres y seis horas, ${ }^{22}$ se observó asociación entre no cumplimiento y menor riesgo de mortalidad a 30 días $(\mathrm{RM}=0.143$, IC $95 \%=0.031-0.659, p=0.013$ ).

\section{Discusión}

Las variables demográficas como el tipo de hospital, la edad, el sexo y el índice de masa corporal no influyeron en la mortalidad, la cual en los pacientes que desarrollaron choque séptico fue mayor que en los pacientes que solo presentaron sepsis., ${ }^{1,2,5}$ Entre las infecciones destacan las del tracto urinario y las neumonías, esta última incrementó el riesgo de mortalidad; un número elevado de pacientes con infección urinaria reportaron cultivos negativos. ${ }^{8,9,19}$ Las puntuaciones medias de las escalas de evaluación de la gravedad (APACHE II, SAPS II, SOFA, qSOFA y MPM0-II) fueron significativamente más altas en los pacientes que fallecieron. ${ }^{2}$

Observamos que el lactato fue significativamente mayor en los no supervivientes. Poco menos de $40 \%$ de los pacientes se sometieron a cultivo para aislamiento microbiológico, sin embargo, el rendimiento no llegó a $20 \%$. Fue alarmante que poco menos de $30 \%$ recibió tratamiento antimicrobiano en la primera hora. Un número reducido de pacientes recibió la cantidad de líquidos recomendada por las guías; ${ }^{22} \sin$ embargo, recibir menos líquidos se asoció a menor mortalidad, probablemente por menor sobrecarga hídrica. 
Tabla 2. Apego a metas de reanimación* en pacientes con sepsis atendidos en servicios de urgencias médicas

\begin{tabular}{|c|c|c|c|c|c|c|c|}
\hline & \multicolumn{2}{|c|}{ Todos los pacientes } & \multicolumn{2}{|c|}{ Sobrevivientes } & \multicolumn{2}{|c|}{ No sobrevivientes } & \multirow[t]{2}{*}{$p$} \\
\hline & $\mathrm{n}$ & $\%$ & n & $\%$ & $\mathrm{n}$ & $\%$ & \\
\hline $\begin{array}{l}\text { Muestreo de lactato } \\
\text { Sí } \\
\text { No }\end{array}$ & $\begin{array}{c}64 / 307 \\
243 / 307\end{array}$ & $\begin{array}{l}20.8 \\
79.1\end{array}$ & $\begin{array}{c}43 / 64 \\
212 / 243\end{array}$ & $\begin{array}{l}67.1 \\
87.2\end{array}$ & $\begin{array}{c}21 / 64 \\
31 / 243\end{array}$ & $\begin{array}{l}32.8 \\
12.7\end{array}$ & 0.0002 \\
\hline $\begin{array}{l}\text { Obtención de cultivos } \\
\text { Sí } \\
\text { No }\end{array}$ & $\begin{array}{c}72 / 307 \\
235 / 307\end{array}$ & $\begin{array}{l}23.4 \\
76.5\end{array}$ & $\begin{array}{c}53 / 72 \\
202 / 235\end{array}$ & $\begin{array}{l}73.6 \\
85.9\end{array}$ & $\begin{array}{c}19 / 72 \\
33 / 235\end{array}$ & $\begin{array}{c}26.3 \\
14\end{array}$ & 0.0162 \\
\hline $\begin{array}{l}\text { Antibiótico en la primera hora } \\
\text { Sí } \\
\text { No }\end{array}$ & $\begin{array}{c}86 / 287 \\
201 / 287\end{array}$ & $\begin{array}{l}29.96 \\
70.03\end{array}$ & $\begin{array}{c}71 / 86 \\
168 / 201\end{array}$ & $\begin{array}{l}82.5 \\
83.5\end{array}$ & $\begin{array}{c}15 / 86 \\
33 / 201\end{array}$ & $\begin{array}{l}17.4 \\
16.4\end{array}$ & 0.055 \\
\hline \multicolumn{8}{|c|}{ Meta de líquidos (mL/kg): $30 \mathrm{~mL} / \mathrm{kg}$ en las primeras tres horas } \\
\hline & $n$ & Media & n & Media & $\mathrm{n}$ & Media & \\
\hline Sí & $38 / 307$ & 39.28 & $24 / 38$ & 38.95 & $14 / 38$ & 39.94 & 0.7263 \\
\hline No & $269 / 307$ & 7.95 & 231/269 & 7.74 & $38 / 269$ & 9.19 & 0.7565 \\
\hline \multicolumn{8}{|c|}{ Apego a las metas en la primera hora } \\
\hline & \multicolumn{2}{|c|}{$\mathrm{n}$} & \multicolumn{2}{|c|}{ n } & \multicolumn{2}{|c|}{$n$} & \\
\hline Sí & \multicolumn{2}{|c|}{$9 / 307$} & \multicolumn{2}{|c|}{$5 / 9$} & \multicolumn{2}{|c|}{$4 / 9$} & 0.038 \\
\hline No & \multicolumn{2}{|c|}{$298 / 307$} & \multicolumn{2}{|c|}{250} & \multicolumn{2}{|c|}{$48 / 298$} & \\
\hline \multirow[t]{2}{*}{$\begin{array}{l}\text { Apego a las metas en las tres } \\
\text { Sí } \\
\text { No }\end{array}$} & \multicolumn{2}{|c|}{$\begin{array}{c}7 / 307 \\
300 / 307\end{array}$} & \multicolumn{2}{|c|}{$\begin{array}{c}3 / 7 \\
252 / 300\end{array}$} & \multicolumn{2}{|c|}{$\begin{array}{c}4 / 7 \\
48 / 300\end{array}$} & 0.013 \\
\hline & \multicolumn{2}{|c|}{ Media } & & & & & \\
\hline Lactato arterial (mmol/L) & & & & & & & 0.003 \\
\hline $\begin{array}{l}\text { Balance hídrico acumulado (m } \\
\text { Primeras } 3 \text { horas } \\
\text { Primeras } 6 \text { horas } \\
\text { Primeras } 24 \text { horas }\end{array}$ & & & & & & & $\begin{array}{l}0.0271 \\
0.0587 \\
0.0969\end{array}$ \\
\hline
\end{tabular}

*Conforme Surviving Sepsis Campaign. ${ }^{21,22}$

Valor de $p$ entre supervivientes y no supervivientes.

Llama la atención que en pocos casos se logró el cumplimiento total de los objetivos de reanimación. ${ }^{21,22}$ La interpretación de este resultado debe tomarse con precaución, ya que el bajo número de pacientes en quienes se cumplieron esas recomendaciones puede sesgar el análisis y denotar resultados que pueden no ser ciertos en la práctica diaria; además, esas recomendaciones fueran diseñados para países de alto ingreso y con enfoque en la unidades de cuidados intensivos, no en los SUM.

\section{Fortalezas y limitaciones}

Este estudio tiene algunas limitaciones:

1. La participación en el estudio fue voluntaria y no estamos seguros de que la muestra sea suficiente o aleatoria y represente a los SUM de México.

2. La participación de hospitales privados fue muy baja y no podemos determinar el efecto en los resultados.

3. Se observó que el examen general de orina se utiliza para diagnosticar infecciones del tracto urinario, lo cual puede llevar al sobrediagnóstico.

4. Algunos SUM participantes carecen de médicos especialistas en medicina de urgencias, otros son hospitales universitarios, algunos tienen un servicio de infectología que pueden influir en el tratamiento antimicrobiano óptimo y otros son hospitales de tercer nivel con excelentes recursos, Desconocemos el impacto de todas estas variables en la calidad de la atención, el cumplimiento 
de los objetivos de reanimación y el manejo al egreso del SUM.

5. Desconocemos la capacitación, actualización, protocolos de atención y calificaciones educativas del personal adscrito a los SUM.

A pesar de lo anterior, se trató de un estudio epidemiológico prospectivo amplio, que proporciona información valiosa sobre la sepsis en los SUM de México que puede servir como base para futuras investigaciones centradas en patógenos, infecciones, factores de riesgo, antimicrobianos, fluidos y calidad de la atención, entre otros temas.

\section{Interpretación}

No hay estudios similares relativos a los servicios de urgencias de Latinoamérica. La prevalencia de $12.9 \%$ de sepsis fue mayor a la indicada en otros estudios realizados en servicios de urgencias de Estados Unidos $(0.64 \% \text {, IC } 95 \%=0.57-0.73 \%)^{9}$ y Reino Unido $(4.3 \% \text {, IC } 95 \%=3.3-5.2 \%)^{7}$ y representó cerca del doble de la observada en UCI de Turquía $(6.9 \%)^{23}$ o la mitad de la registrada en $\mathrm{UCl}$ de Brasil $(30.2 \%) .{ }^{24}$ En cuanto a las variables demográficas y el sitio de infección, no encontramos diferencias importantes, si bien se registró alta frecuencia de infecciones del tracto urinario en comparación con otras series. 7,9,10,23-25

Observamos alarmante falta de recursos en relación con la medición de lactato, exámenes de laboratorio, cultivos, acceso a antibióticos en un tiempo aceptable y cumplimiento de los objetivos de reanimación. Encontramos que los pacientes que no recibieron reanimación con líquidos de acuerdo con las recomendaciones internacionales ${ }^{21,22}$ tuvieron menor mortalidad. Si bien se ha demostrado que la reanimación hídrica oportuna se asocia a menor mortalidad, ${ }^{26}$ los efectos nocivos de la sobrecarga de líquidos también son un factor de riesgo importante para mayor mortalidad. ${ }^{27-30}$

Creemos que existen diversos factores no medidos en este estudio que afectan la atención de los pacientes con sepsis en México: la atención en consultorios anexos a farmacias puede reducir las posibilidades de prevención y aumentar la prescripción inadecuada de antimicrobianos; ${ }^{31}$ la saturación de los SUM debido a la falta de procesos de triaje, la referencia tardía y la insuficiencia de personal o recursos, ${ }^{32,33}$ la restricción en el acceso a los servicios de laboratorio de microbiología en ciertos horarios; la escasa referencia a la unidad de cuidados intensivos (menos de $8 \%$ de los pacientes en esta cohorte); y el desconocimiento de las guías nacionales sobre sepsis ${ }^{34-36}$ y otros documentos relacionados ${ }^{37-40} \mathrm{o}$ la preferencia por el empleo de guías internacionales.

En conclusión, encontramos que la prevalencia y mortalidad de lo pacientes con sepsis en los SUM de México fueron elevadas. El acceso limitado a los recursos puede ser un factor de riesgo asociado a la mortalidad. Se necesitan programas de capacitación y vigilancia para mejorar la calidad de la atención, lo cuales busquen reducir la carga ${ }^{41}$ que representa la sepsis para el sistema de salud de México.

\section{Contribuciones de los autores}

Luis A. Gorordo-Delsol tuvo acceso completo a todos los datos y se responsabiliza por la integridad de estos y la precisión de su análisis; además, junto con Graciela Merinos-Sánchez y Ma. Angélica Morales-Segura participó en el análisis estadístico de los datos. Todos los autores participaron directa y sustancialmente en el diseño e interpretación de resultados y contribuyeron sustancialmente en la redacción, revisión y aprobación del manuscrito final.

\section{Conflicto de intereses}

No hay conflictos de intereses que reportar.

\section{Financiamiento}

No se recibió ninguna subvención para este estudio.

\section{Responsabilidades éticas}

Protección de personas y animales. Los autores declaran que los procedimientos seguidos se apegaron a las normas éticas del comité de experimentación humana responsable y a los lineamientos de la Asociación Médica Mundial y la Declaración de Helsinki.

Confidencialidad de los datos. Los autores declaran que siguieron los protocolos de su centro de trabajo sobre la publicación de datos de pacientes.

Derecho a la privacidad y consentimiento informado. Dada la naturaleza de la investigación, no fue necesaria la obtención de consentimiento informado.

\section{Bibliografía}

1. Singer M, Deutschman CS, Seymour CW, Shankar-Hari M, Annane D, Bauer M, et al. The Third International Consensus Definitions for Sepsis and Septic Shock (Sepsis-3). JAMA. 2016;315:801-810. 
2. Seymour CW, Cinvent XL, Iwashyma TJ, Brunkhorst FM, Rea TD, Sche$\operatorname{rag} A$, et al. Assessment of clinical criteria for sepsis: for the Third International Consensus Definitions for Sepsis and Septic Shock (Sepsis-3). JAMA. 2016;315:762-774.

3. Donnelly JP, Safford MM, Shapiro NI, Baddley JW, Wang HE. Application of the Third International Consensus Definitions for Sepsis (Sepsis-3) classification: a retrospective population-based cohort study. Lancet Inf Dis. 2017:17:661-670.

4. Machado FR, Nsutebu E, AbDulaziz S, Daniels R, Finfer S, Kissoon N, et al. Sepsis 3 from the perspective of clinicians and quality improvement initiatives. J Crit Care. 2017:40:315-317.

5. Shankar-Hari M, Phillips GS, Levy ML, Seymour CW, Liu VX, Deutschman CS, et al. Developing a new definition and assessing new clinical criteria for septic shock for the Third International Consensus Definitions for Sepsis and Septic Shock (Sepsis-3). JAMA. 2016;315:775-787.

6. Ibrahim I. Sepsis: the need for epidemiological studies in the emergency department. J Emer Med. 2014;46:e93-e94.

7. Cowan SL, Holland JAA, Kane AD, Frost I, Boyle AA. The burden of sepsis in the emergency department: an observational snapshot. Eur Emerg Med. 2015;22:363-365.

8. Yu CW, Chang SS, Lai CC, Wu JY, Yen DW, Lee MG, et al. Epidemiology of emergency department sepsis: a national cohort study between 2001 and 2012. Shock. 2019;51:619-624.

9. Wang HE, Joes AR, Donnelly JP. Revised national estimates of emergency department visits for sepsis in the United States. Crit Care Med. 2017:45:1443-1449.

10. Wang HE, Shapiro NI, Angus DC, Yealy DM. National estimates of severe sepsis in United States emergency departments. Crit Care Med. 2007:35:1928-1936.

11. Angus DC, Linde-Zwirble WT, Lidicker J, Clermont G, Cardillo J, Pinsky MR. Epidemiology of severe sepsis in the United States: analysis of incidence, outcome, and associated cost of care. Crit Care Med. 2001;29:1303-1310.

12. Lide-Zwirble WT, Angus DC. Severe sepsis epidemiology: sampling, selection, and society. Crit Care. 2004;8:222-226.

13. van Dillen J, Zwart J, Schutte J, van Roosmalen J. Maternal sepsis: epidemiology, etiology and outcome. Curr Opin Infect Dis. 2010;23:249-254.

14. Watson RS, Carcilo JA. Scope and epidemiology of pediatric sepsis. Pediatr Crit Care Med. 2005;6:S3-S5

15. Rhodes A, Phillips G, Beale R, Cecconi M, Chiche JD, de Backer D, et al. The Surviving Sepsis Campaign Bundles and outcome: results from the International Multicentre Prevalence Study on Sepsis (the IMPreSS study). Intensive Care Med. 2015;41:1620-1628.

16. Vincent JL, Rello J, Marchall J, Silva E, Anzueto A, Martín CD, et al. International study of the prevalence and outcomes of infection in intensive care units. JAMA. 2009:302:2323-2329.

17. Cabrera A, Laguna G, López G, Villagómez A, Méndez R, Guzmán R. Mecanismos patogénicos en sepsis y choque séptico. Med Int Mex. 2008;24:38-42

18. Carrillo R, Carrillo JR, Carrillo LD. Estudio epidemiológico de la sepsis en unidades de terapia intensiva mexicanas. Cir Cir. 2009;77:301-308.

19. Gorordo-Delsol LA, Mérida-García JA, López-Gómez A. Sepsis: más allá de la enfermedad. Arch Med Urg Mex. 2014:6:12-16.

20. Von Elm E, Altman DG, Egger M, Pocock SJ, Gøtzsche PC, Vanderbroucke JP, et al. The Strengthening the Reporting of Observational Studies in Epidemiology (STROBE) statement: guidelines for reporting observational studies. J Clin Epidemiol. 2008;61:344-349.

21. Levy MM, Evans LE, Rhodes A. The Surviving Sepsis Campaign Bundle: 2018 update. Intensive Care Med. 2018;46:997-1000.
22. Rhodes A, Evans LE, Alhazzani W, Levy M, Massimo A, Ferrer R, et al. Surviving Sepsis Campaign: International Guidelines for Management of Sepsis and Septic Shock: 2016. Intensive Care Med. 2017;43:304-377.

23. Baykara N, Akalın H, Arslantaş MK, Hancı V, Cağlayan C, Kahveci F, et al. Epidemiology of sepsis in intensive care units in Turkey: a multicenter, point-prevalence study. Crit Care. 2018;22:93.

24. Machado FR, Cavalcanti AB, Bozza FA, Ferreira EM, Angotti Carras FS, Sousa JL, et al. The epidemiology of sepsis in Brazilian intensive care units (the Sepsis PREvalence Assessment Database, SPREAD): an observational study. Lancet Infect Dis. 2017;17:1180-1189.

25. Rezende E, Silva JM, Isola AM, Campos EV, Amendola CP, Almeida SL. Epidemiology of severe sepsis in the emergency department and difficulties in the initial assistance. Clinics (Sao Paulo). 2008;63:457-464.

26. Leisman D, Wie B, Doerfler M, Bianculliu A, Ward MF, Akerman M, et al. Association of fluid resuscitation initiation within 30 minutes of severe sepsis and septic shock recognition with reduced mortality and length of stay. Ann Emerg Med. 2016;68:298-311.

27. Boyd JH, Forbes J, Nakada TA, Walley KR, Russel JA. Fluid resuscitation in septic shock: a positive fluid balance and elevated central venous pressure are associated with increased mortality. Crit Care Med. 2011;39:259-265.

28. Mithell KH, Carlbom D, Cadwell E, Leary PJ, Himmelfarb J, Houg CL. Volume overload: prevalence, risk factors, and functional outcome in survivors of septic shock. Ann Am Thorac Soc. 2015;12:1837-1844.

29. De Oliveira FS, Freitas FG, Farreira EM, de Castro I, Bafi AT, de Azevedo LC, et al. Positive fluid balance as a prognostic factor for mortality and acute kidney injury in severe sepsis and septic shock. J Crit Care. 2015;30:97-101.

30. Sirvent JM, Ferri C, Baró A, Murcia C, Lorencio C. Fluid balance in sepsis and septic shock as a determining factor of mortality. Am J Emerg Med. 2015;33:186-189.

31. Dreser A, Wirtz VJ, Corbett KK, Echániz G. Uso de antibióticos en México: revisión de problemas y políticas. Salud Publica Mex 2008;50:S480-S487.

32. Castañón-González JA, Barrientos-Fortes T, Polanco-González C. Reflexiones sobre el proceso de atención en los servicios de urgencias. Rev Med Inst Mex Seg Soc. 2016;54:376-379.

33. Gorordo-Delsol LA, Merinos-Sánchez G. Urgencias: ¿dónde están los especialistas? Rev Med Inst Mex Seg Soc. 2017;55:7-8.

34. Diagnóstico y tratamiento de sepsis y choque séptico en pacientes de 1 mes a 18 años de edad. México: Secretaría de Salud; 2008.

35. Diagnóstico y tratamiento de sepsis grave y choque séptico en el adulto. México: Secretaría de Salud; 2009.

36. Secretaría de Salud. Guía de Práctica Clínica: prevención, diagnóstico y tratamiento de la sepsis materna. México: Secretaría de Salud; 2018.

37. Norma Oficial Mexicana NOM-045-SSA2-2005, para la vigilancia epidemiológica, prevención y control de las infecciones nosocomiales. México: Diario Oficial de la Federación; 2009.

38. Acuerdo por el que se declara la obligatoriedad de la Estrategia Nacional de Acción contra la Resistencia a los Antimicrobianos. México: Diario Oficial de la Federación; 2018.

39. Reinhart K, Daniels R, Kisoon N, Machado FR, Schachter RD, Finfer S. Recognizing sepsis as a global health priority - A WHO resolution. N Engl J Med. 2017;377:414-417

40. Azevedo LCP, Cavalcanti AB, Lisboa T, Dal Pizzol F, Machado FR. Sepsis in an important healthcare burden in Latin America: a call to action! Rev Bras Ter Intensiva. 2018;30;402-404.

41. Gorordo-Delsol LA. Sepsis: the hidden enemy between the lines. Rev Med Inst Mex Seguro Soc. 2017;55:423. 